Cahiers de littérature orale

$70 \mid 2011$

L'adresse indirecte ou la parole détournée

\title{
Chanter la querelle : le séga de Ti Frèr
}

(Île Maurice, début du xx ${ }^{\mathrm{e}}$ siècle)

\section{Caroline Déodat}

\section{(2) OpenEdition}

Journals

Édition électronique

URL : https://journals.openedition.org/clo/1334

DOI : 10.4000/clo.1334

ISSN : 2266-1816

Éditeur

INALCO

\section{Édition imprimée}

Date de publication : 30 décembre 2011

Pagination : 119-138

ISBN : 978-2-85831-202-3

ISSN : 0396-891X

Référence électronique

Caroline Déodat, «Chanter la querelle : le séga de Ti Frèr », Cahiers de littérature orale [En ligne], 70 | 2011, mis en ligne le 18 mars 2013, consulté le 30 juin 2021. URL : http://journals.openedition.org/ clo/1334; DOI : https://doi.org/10.4000/clo.1334

Ce document a été généré automatiquement le 30 juin 2021.

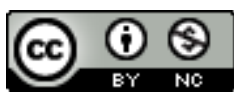

Cahiers de littérature orale est mis à disposition selon les termes de la Licence Creative Commons Attribution - Pas d'Utilisation Commerciale 4.0 International. 


\section{Chanter la querelle : le séga de $\mathrm{Ti}$ Frèr}

(île Maurice, début du Xx ${ }^{\mathrm{e}}$ siècle)

\section{Caroline Déodat}

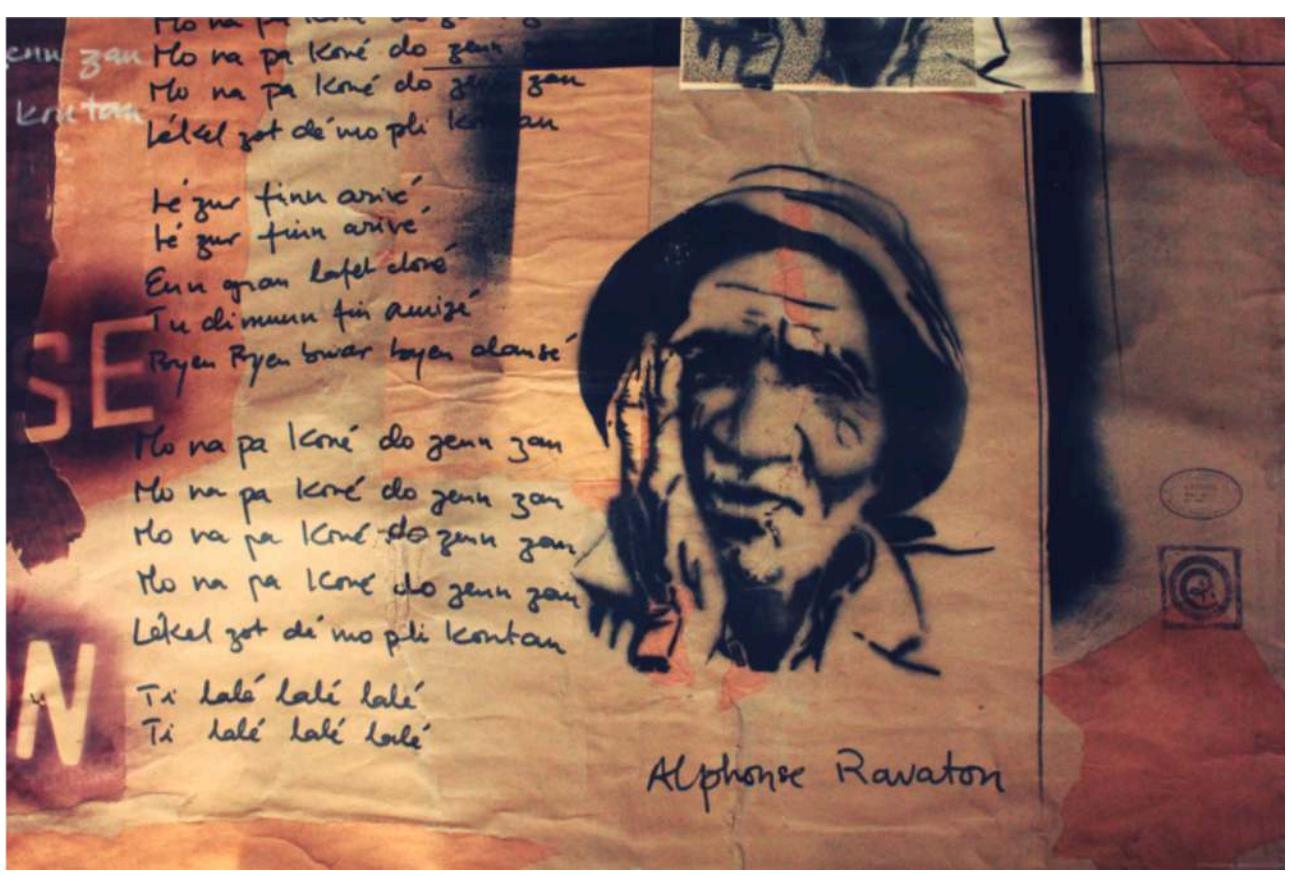

(c) Firoz Ghanty, TI FRER IV (détail)

(Collection privée Catherine Servan-Schreiber)

1 Forme poétique et musicale dansée aujourd'hui très vivante à l'île Maurice, le séga est né dans le contexte douloureux de la colonisation esclavagiste. Dans son histoire et sa création, il a suivi un processus de créolisation impliquant divers apports et influences ${ }^{1}$. Tradition héritée des esclaves ${ }^{2}$ déportés sur la colonie entre les $\mathrm{XVII}^{\mathrm{e}}$ et XIX ${ }^{e}$ siècles, le séga a continué de s'exercer après l'abolition. 
2 La colonisation esclavagiste qui a tant marqué l'histoire de l'île Maurice a laissé des empreintes persistantes dans l'organisation de la société en construisant des espaces de domination et de subalternité. La population mauricienne a ainsi été répartie en plusieurs groupes religieux et ethniques ${ }^{3}$ parmi lesquels se distinguent les Créoles ${ }^{4}$. La communauté créole mauricienne a été assimilée aux descendants des esclaves africains malgaches, et a été stigmatisée comme tels ${ }^{5}$. Le séga, présenté comme l'héritage culturel des esclaves noirs, et par extension, des Créoles, a longtemps été considéré comme une pratique indigne et, de fait, exclu des espaces officiels. Il s'est constitué comme une pratique de transgression, outrepassant les tabous sociaux de la société mauricienne qui tient pour honteux tout ce qui rappelle l'esclavage. Au début du $\mathrm{xx}^{\mathrm{e}}$ siècle, le séga s'exerce ainsi dans une certaine marginalité.

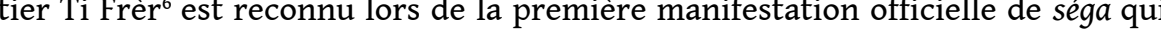
se tint le 24 octobre $1964^{7}$ et qui prit la forme d'un concours entre des ségatiers venus de toute l'île. Ti Frèr devient une figure emblématique ${ }^{8}$ du séga mauricien à partir de 1965 contribuant ainsi en partie à la dimension nationale de cette pratique. Auparavant, il est une figure locale et chante pour la collectivité rurale de Quartier militaire, dans des ambiances festives, à l'occasion de bals les samedis soirs ou de pique-niques au moment de la chasse. Ti Frèr ne vit pas de sa musique, mais subsiste grâce à des travaux divers. Il est entre autres chauffeur de bus, un métier qui lui permet d'observer ses congénères dont il chante les histoires dans ses ségas. Le ségatier Ti Frèr s'inspire de séquences de vie réelle, d'événements vécus par des individus existants ou ayant existé ${ }^{9}$ qu'il met en paroles et en musique.

Dans ses ségas, Ti Frèr interprète un ou plusieurs personnages dans de véritables mises en scène de fiction. Il s'agit surtout de scènes de querelle sur des faits du quotidien, qui vont de la discorde amicale à la dispute conjugale, de la misère du chômage à celle de l'alcoolisme et de la violence. Les critiques et les commentaires hostiles qui se déploient au sein de la fiction couvrent des intentions sociales : rappeler de manière indirecte des règles de bonne conduite à l'ensemble de la communauté présente lors du séga. Dans les ségas de Ti Frèr, l'opération rituelle, qui s'exerce à travers le chant, la musique et la danse, se situe sur le comportement d'un individu.

\section{Faire un séga}

5 Le terme séga est "plurisémantique » (Desrosiers, 2004, 257). Il désigne à la fois un genre, par exemple le "séga typique ", le "séga engagé » ou plus récemment le «séga Bollywood » (Servan-Schreiber, 2010). Il peut se référer également à la danse seule, ou à la musique seule, en même temps qu'à l'événement lui-même qui se constitue à la fois du chant, de la musique et de la danse. La description du séga qui va suivre est le résultat d'un travail ethnographique en cours. Il s'agit d'une proposition sur ce que pouvaient constituer les performances ${ }^{10}$ de ségas de Ti Frèr au début du $\mathrm{xx}^{\mathrm{e}}$ siècle.

6 La manifestation du séga réunit le ségatier, les musiciens ${ }^{11}$ et les membres de l'assemblée qui prennent également part à la performance. L'assemblée s'adapte et s'installe en fonction des lieux, laissant suffisamment de place pour permettre l'exécution de la danse. Le groupe ne se compose pas de danseurs attitrés, car la danse est pratiquée par les membres de l'assemblée présente. 
7 Au début, les musiciens se préparent, précisément ceux qui jouent de la ravane. Ils doivent en effet chauffer la peau du tambour au-dessus du feu pour que celle-ci soit bien tendue et produise le son attendu. Cette manipulation effectuée, le séga peut commencer, il va durer tant que résonne le tambour, car il vient un moment où la membrane de la ravane se distend et perd sa sonorité. Alors, les musiciens, le ségatier et les danseurs ne peuvent que cesser toutes activités, et attendre que les ravaniers chauffent de nouveau la peau du tambour pour reprendre un autre séga.

8 Le ségatier chante de sa voix rauque les paroles, les répète à l'envi et ponctue souvent son chant d'onomatopées qui vont contribuer, au même titre que les battements des instruments, au rythme intense de la danse. Les membres de l'assemblée qui se trouvent en face de lui prennent part au mouvement d'intensification initié par le ségatier. Ceux-ci participent aux pulsations du séga en frappant des mains de manière discontinue. Ils peuvent en outre chanter, notamment en reprenant les paroles, mais le plus souvent ils se chargent de vocaliser par des onomatopées comme " olalelalolo ». Ils agissent en fait comme une caisse de résonance en amplifiant de manière sonore les chants du ségatier.

Les participants viennent à un séga avec leurs habits de tous les jours. Il n'y a pas de costumes particuliers à revêtir. Pourtant le séga instaure une temporalité et des valeurs sensiblement différentes de celles du quotidien, il est une fête, au sens anthropologique du terme.

La forme des ségas de Ti Frèr ne suit pas des règles fixes et unitaires pouvant correspondre à une structure musicale précise ou à une versification donnée. Toutefois il existe des parcours obligés que chacun, dans l'assemblée, saura reconnaître comme «un bon séga ». Parmi les compositions de Ti Frèr, la musicologue Claudie Ricaud (1993) dégage deux formes distinctes de séga : un premier type qu'elle nomme le « séga é-la-é » ou " séga-laé " ${ }^{12}$ - intitulé qui rappelle les onomatopées chantées en fin de séga pour stimuler la danse, et un second, le « séga final », qui tirerait quant à lui son nom du quadrille européen ${ }^{13}$. L'organisation du "séga-laé» suit une progression qui va crescendo et dont le but est la réunion du chant, de la musique et de la danse. L'interrelation entre les paroles, la musique et la danse, qui se produisent ensemble et s'affectent les unes les autres, aboutit à une explosion finale où chacun participe à la montée en puissance, les danseurs étant excités et encouragés par les répétitions verbales et vocales. Nous pouvons observer ces principes de composition du "séga-laé » à travers le séga « Roseda».

\section{Roseda}

Gra maten mo levé

Mo bure mo al travay

Sito dizer li soné

Kuma mo usi return dan lakur

Kuma mo return dan lakur

Mo truv mo piti bor difé

Ape badiné bor difé

Avek ponyé lasann dan lamen

Parol mo dir piti la

Akot to mama finn alé

Mama finn al labutik

Labutik sinwa kor de gard 
Mo pik simen labutik

Mo tann diskité ar sinwa

Diskite ar sinwa

Avek so ver larak dan lamen

Mo kryé li Roseda da Roseda

Zoli ti fam kuma twa

Zoli fam kuma twa

To zenes fini dan labutik

Kuma lakoler pran mwa

Enn lakoler pran mwa

Kuma lakoler pran mwa

Mo ris li mo amenn dan lakur

Roseda

Je me réveille de bon matin

Je pars à la hâte pour aller au travail

Aussitôt que dix heures sonnent

Je quitte l'usine et rentre chez moi

Je rentre chez moi

Je trouve mon enfant près du feu

Il joue près du feu

Une poignée de cendres dans la main

Je demande à mon enfant

Où est allée ta maman

Maman est allée à la boutique

La boutique du chinois à Corps de garde

Je suis le chemin vers la boutique

J'entends qu'elle parle avec le boutiquier chinois

Qu'elle parle avec le boutiquier chinois

Avec son verre d'arack dans la main

Je crie Roseda, Roseda

Une jolie femme comme toi

Une jolie femme comme toi

Tu passes ta vie dans la boutique

La colère me prend

La colère m'envahit

La colère me prend

Je la saisis et l'emmène à la maison

11 La forme strophique du séga "Roseda » - reproduite ici dans la transcription - est particulièrement mise en évidence dans l'élaboration de la musique et du chant. En fait, l'énoncé de la strophe correspond à une intervention autonome du ségatier, qui se fait sans la musique. L'intervention achevée, le rythme de la ravane surgit puis cesse. Le ségatier chante d'une voix rauque et gutturale la deuxième strophe en silence. Cette organisation par intermèdes, qui fait alterner chant et musique et qui correspond à la deuxième partie du "séga-laé", se poursuit ainsi jusqu'à la sixième strophe. La troisième partie débute quand le chant et la musique interviennent ensemble. Aussitôt, les membres de l'assemblée vocalisent, le rythme du triangle se mêle à celui de la maravane et de la ravane, alors que le ségatier entame la reprise des premières paroles 
du séga. Il y a ainsi dans les ségas de Ti Frèr une réciprocité entre la poésie chantée, la musique et la danse.

Dans « Roseda », les paroles font intervenir des jeux de langage qui favorisent des effets phoniques et soutiennent le rythme des corps et des airs chantés, conjointement aux percussions et à la mélodie. Ces procédés poétiques apportent une sorte de scansion par effets de renforcement des battements et des pulsations de la cadence du séga, par des jeux d'homophonie et de rimes. Dans les deuxième, quatrième, cinquième et sixième strophes de "Roseda", l'on peut observer des rimes embrassées qui sont le fait de répétitions d'une même phrase dans l'unité de la strophe. De même, l'on peut noter les anaphores « mo » et « zoli », respectivement dans les quatrième et cinquième strophes. Et enfin des allitérations en $[\mathrm{k}],[\mathrm{p}],[\mathrm{m}],[\mathrm{z}]$ et $[\mathrm{s}]^{14}$ tout au long du séga. Le matériau verbal étant court et bref, les répétitions servent encore à faire durer la performance. Le chanteur répète les paroles du séga à plusieurs reprises, ce qui va encourager le chœur à répondre et les danseurs à poursuivre leurs pas. Le verbal est également intimement lié à la gestuelle.

Le séga implique une danse en couple. Celle-ci s'exécute dans des élans d'attractions et de repoussements, comme un jeu de séduction où l'homme et la femme dansent en se provoquant l'un l'autre. Le couple se tient d'abord face à face et se déplace en tournant par petits pas latéraux accompagnés de déhanchements. Dans ces moments, le haut du corps reste relativement immobile alors que les jambes sont constamment pliées et que les hanches s'agitent sans cesse ${ }^{15}$. Les déhanchements exécutés par la femme sont les plus spectaculaires par leur rapidité et les plus marqués dans leurs ondulations. La danse se poursuit par des déplacements de l'homme qui tourne autour de la femme, ou l'inverse, chacun s'éloignant et se rapprochant de l'autre en se frôlant. La femme danse en agitant sa jupe, l'homme la suit en écartant parfois les bras. La danse se complexifie quand un autre homme passe entre le premier danseur et sa partenaire pour s'en emparer: on dit ainsi qu'il "coupe». La femme change alors de partenaire et commence une autre danse, jusqu'au moment où le couple sera de nouveau coupé par un autre danseur. La danse se pratique dans la décontraction et l'effervescence, les corps des danseurs sont légèrement dénudés. Les hommes peuvent se mouvoir torse nu tandis que les femmes ont les jambes et les bras nus le plus souvent.

Le mouvement des corps est encouragé par le rythme ternaire des tambours. Sollicité par les danseurs, le corps ${ }^{16}$ constitue également un support musical et rythmique, les participants battent des mains, mais frappent aussi leur corps dans son ensemble. À la fois instrument de percussion et de présence, le corps est aussi un référent de la parole poétique. Il est évoqué explicitement dans des appels du ségatier aux mouvements des reins et des hanches. La communication qui lie celui-ci aux membres de l'assemblée, et en particulier aux danseurs, fait allusion au corps. Il répète des onomatopées (olalelolo) qui tonifient les danseurs, mais aussi des phrases courtes et percutantes comme : mo vini (je viens), doucement dans les reins, donne to le rein (donne tes reins). Ces références à certaines parties du corps, et en particulier l'appel à « donner » ses hanches et ses reins constituent très probablement des allusions sexuelles. Maurice Coyaud qui a étudié « La transgression des bienséances dans la littérature orale ", nous dit que les tabous sexuels peuvent être «bafou[és] clairement, ou par allusion. Le second procédé [n'étant] pas le moins puissant» (Coyaud, 1980:386). Dans les paroles et la danse de séga, la transgression du tabou sexuel s'effectue de manière implicite dans une communication 
qui lie le ségatier aux danseurs. Dans les ségas de Ti Frèr, la poésie est véritablement incarnée (embodied) dans la danse autant qu'elle est figurée par le corps.

Les paroles de tels ségas tiennent dans un équilibre entre signifiant et signifié, entre son et sens, ce qui redouble l'impact du message et l'interaction du ségatier avec les participants. Ces derniers prennent part à l'événement à travers le chant et la danse dans une communication rythmée et chantée qui les lie au ségatier Ti Frèr.

\section{Une communication indirecte}

\section{Une stratégie de l'énonciation}

16 La situation d'énonciation des ségas de Ti Frèr implique un jeu relationnel pluriel dans lequel plusieurs voix se font entendre. Le ségatier-locuteur d'une part, en tant que sujet parlant, existe de manière effective, tout comme les membres de l'assemblée, qui répondent en vocalisant ou en chantant certaines des paroles de ce dernier. Dans ses ségas, Ti Frèr chante rarement en son nom propre, sauf dans les moments où il interpelle les participants pour qu'ils chantent ou dansent (« krié »...). Il incarne le plus souvent des personnages, derrière lesquels lui-même s'efface, en leur prêtant sa voix. À l'intérieur de l'acte énonciatif du locuteur principal, il existe ainsi une seconde parole qui correspond à celle d'un personnage de fiction. Le locuteur subsume donc différentes positions dont l'indice de son dédoublement est le changement de référent du pronom je.

17 La situation discursive de tels ségas est complexe, car le ségatier-locuteur actualise parfois plusieurs personnages, que nous appellerons énonciateurs ${ }^{17}$. Dans le cadre de la fiction, l'énonciateur s'adresse à un autre personnage, également incarné par le ségatier-locuteur, et que nous appellerons l'énonciataire. Celui-ci est parfois amené à répondre dans de véritables situations de dialogue. Cette situation discursive originale, dans laquelle énonciateur et énonciataire interagissent, est rendue possible par le ségatier-locuteur qui met en voix et en scène ce dialogue. Il fait alors exister deux personnages dans un même séga. Cette configuration se retrouve par exemple dans les ségas « Fidelia » ou encore "Roseda » ${ }^{18}$. Cette stratégie énonciative opère un véritable renversement des identités puisqu'il revient au ségatier-locuteur d'endosser plusieurs masques.

18 Les membres de l'assemblée tiennent une place importante dans la communication des ségas de Ti Frèr. Le ségatier s'adresse de manière directe aux participants, par des impératifs ("cozé») pour les encourager à chanter, ou par des onomatopées pour stimuler la danse. Le public, qui se constitue en chœur, se met à lui répondre en reprenant les paroles et en vocalisant des syllabes non lexicalisées. Cette forme dialogale particulière, dans laquelle se distribue la parole sous forme de réponses, qui unit le ségatier-locuteur et les membres de l'assemblée, s'apparente à ce que l'ethnomusicologie nomme une forme responsoriale.

19 Ainsi, dans les ségas de Ti Frèr plusieurs situations de communication apparaissent et se démultiplient en strates énonciatives emboîtées les unes dans les autres. Le système de communication dans lequel échangent les personnages (l'énonciateur à l'énonciataire) prend place à l'intérieur d'un autre procédé de communication, qui unit le ségatier aux participants. Deux cadres de compréhension s'enchevêtrent : la fiction et la réalité. Dans notre schéma, nous avons donc pris le soin de différencier le système 
de communication réel, référentiel (en pointillés) du système de communication « fictif» (en noir).

Schéma 1 : Le système de communication du séga

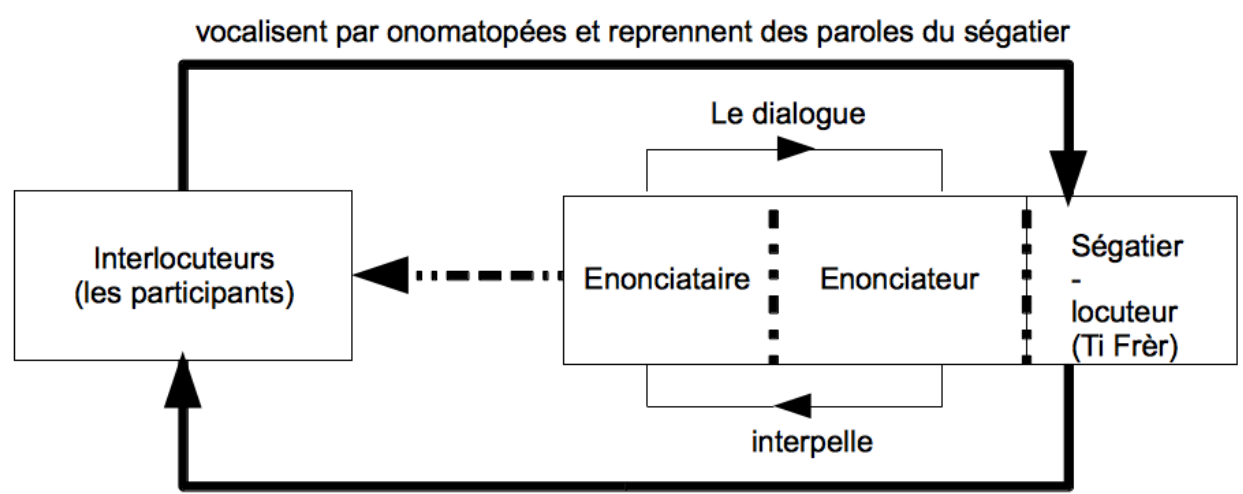

s'adresse aux participants par onomatopées et appels directs

\section{La mise en scène d'une fiction}

Dans la situation de communication des ségas de Ti Frèr, l'instance énonciative principale se cache derrière plusieurs personnages, impliquant divers effets de dramatisation, ce que nous pouvons observer dans « Fidelia ».

\section{Fidelia}

Fidelia do mo fam

Depi ki nu finn marye

Depi ki nu finn marye

Zame ki twa pran lespri

Mo desann mo al anvil mo aste zoli rob pu twa

Zoli rob pu twa avek zano loray lor la

Fidelia Fidelia

Parol mo koz avek twa

Parol mo koz ansam twa

Tusula finn fann partu

Donn mwa le tan ti mama

Les mo tir mo sang dan lerin

Tir mo sang dan lerin

Apre pangar twa truv mwa mesan

Li kry Victorin, do mo mari

Napa tap mwa dan nwar-nwar

Pa tap mwa dan nwar-nwar

Zanfan de zimo ki dan mo vant

Zanfan de zimo ki dan mo vant

Piti lezitim dan lebra

Pa tap mwa dan nwar-nwar

Zanfan de zimo ki dan mo vant 


\section{Fidelia}

Fidelia toi ma femme

Depuis que nous sommes mariés

Depuis que nous sommes mariés

Tu n'en as jamais eu conscience

Je vais en ville, je t'achète une jolie robe

Une jolie robe pour toi et des boucles d'oreille en or

Fidelia Fidelia

Ce que je t'ai dit

Ce que je t'ai dit (à toi seulement)

Tout a été répété

Donne moi un instant p'tite maman

Laisse-moi retirer ma ceinture

Retirer ma ceinture

Après attention tu me trouves méchant

Elle crie Victorin toi mon mari

Ne me bats pas dans le noir

Ne me bats pas dans le noir

Je porte des jumeaux dans mon ventre

Des jumeaux dans mon ventre

Et ton enfant légitime dans mes bras

Ne me bats dans le noir

Je porte des jumeaux dans mon ventre

21 Dans «Fidelia » le cadre du dialogue est tout de suite compréhensible par l'assemblée.

Dès la première strophe, le contexte d'une querelle conjugale est explicité : «Depuis que nous sommes mariés tu n'en as jamais eu conscience » (Depi ki nu finn marye zame ki twa pran lespri). Deux protagonistes sont identifiés, une femme nommée Fidelia et son mari Victorin. La situation de dialogue entre ces deux personnages est immédiatement amorcée par une interpellation : "Fidelia toi ma femme " (Fidelia do mo fam). Cela pour insister sur le fait que c'est le mari qui parle. Les interpellations directes à Fidelia se poursuivent soit par des vocatifs «Fidelia, Fidelia » soit par des appels dirigés à son attention ainsi que l'attestent les six occurrences du pronom «twa». Au début, le mari est le seul mis en scène, il monopolise la parole. L'utilisation d'indices énonciatifs de la première personne qui se référent à l'énonciateur en atteste : "Je descends en ville » (Mo desann mo al anvil) ou encore : "Donne-moi un instant p'tite maman » (Donn mwa le tan ti mama). L'intervention du second personnage, dans la prise en charge effective de la parole, se produit aux deux tiers du séga. Par la suite, le personnage Fidelia possède l'entière responsabilité de la parole, elle s'adresse alors à son mari et le supplie de ne pas la châtier. Ce changement de la parole est explicité par le ségatier-locuteur dans une sorte de narration au discours rapporté : "Elle crie Victorin ». Une telle précision n'est pourtant pas systématique, et il arrive que le ségatier ne chante pas ces paroles. En effet, selon les transcriptions, cet énoncé n'apparaît pas toujours. Un fait lié à l'oralité qui démontre combien l'interprétation du ségatier est importante, sa gestuelle et sa voix doivent être suffisamment efficaces pour que les membres de l'assemblée comprennent le changement de la prise de parole entre les différents personnages. Le ségatier est ainsi indissociable d'une dimension fictionnelle, des attributs et des effets que cette fiction implique : le masque et le travestissement des identités. 
Dans les ségas de Ti Frèr, la dramatisation agit tout particulièrement venant ainsi renforcer l'interactivité avec l'assemblée. Les effets de dramatisation privilégient notamment la confusion d'espaces et de temps entre le réel et la fiction. Les histoires racontées dans les ségas de Ti Frèr sont inspirées de situations réelles qui ont existé à un moment passé, mais qui sont jouées et chantées «comme si » elles étaient vécues dans le présent. Dans «Fidelia », le temps grammatical utilisé ne prend pas en compte le moment pendant lequel s'est produit l'événement. L'utilisation du présent et du futur proche: «Laisse-moi retirer ma ceinture / Après attention tu me trouves méchant " (Les mo tir mo sang dan lerin / Apre pangar twa truv mwa mesan) instaure un parallèle entre la fiction et le réel. L'ancrage dans l'instant est par ailleurs renforcé par l'emploi de l'impératif, « Ne me tape » (Pa tape $m w a)$, qui vient renforcer l'actualisation dans le présent d'une situation passée. La confusion opère également au niveau des espaces, car le lieu qui accueille les participants de la performance correspond au lieu où se situe l'action de la fiction. Dans les deux cas, il s'agit d'un lieu privé, en l'occurrence la cour ou bien la case. L'ancrage spatial réel se confond donc avec celui de l'histoire, ce qui renforce l'effet d'actualisation. En fait, le contexte spatio-temporel de l'histoire racontée se confond avec le contexte spatio-temporel réel de sorte que leur superposition crée un effet de théâtralisation où le réel semble servir de décor à la fiction tandis que la fiction vient nourrir le réel. Cet effet dramatique est encore renforcé par une confusion entre privé et public. Dans ce séga, ainsi que dans « Roseda » ou "Charlie", ce qui appartient à la sphère intime est exposé à l'ensemble de l'assemblée. Une telle situation, très proche du commérage (lapalab en créole mauricien) vient rappeler justement cette expression populaire mauricienne: Kozé souvan vinn séga, ce qui signifie littéralement "faire des palabres devient séga", autrement dit : si l'on fait des commérages, cela sera su de tous (Carpooran, $2011: 9$ ). Il est particulièrement intéressant de constater que, dans l'imaginaire collectif, le ragot est associé au séga.

\section{Pour quels destinataires?}

Les ségas de Ti Frèr privilégient des situations d'appels et, dans le cas du dialogue, de réponses, par un langage qui s'exprime avant tout à travers l'exhortation. C'est un chant dans lequel se multiplient les impératifs, les ordres et les injonctions ${ }^{19}$. La parole s'exprime au sein d'un contexte railleur, dans le détour et la subversion, le comique et la satire. "Charlie» par exemple, mais aussi les deux ségas présentés plus haut, illustrent bien ces conduites verbales.

\section{Charlie}

Charlie 0 Charlie

Aret bwar, aret bwar divin banann

To gagn kinz su to al manz enn grog dan to manyer

Sinapa to perdi lavi sa pov fam-la

Dan divin banann tuzur ena bebet sizo

Bebet sizo me mo krwar li a pik to lagorz

Charlie, Charlie, aret bwar divin banann

Fer mwa sote Kedu samdi mo pay

twa na pa kapav

Mo seye Kedu samdi mo pay twa na pa kapav 
Les mo monte Kedu samdi mo pay twa napa kapav

Les mo seye (baye) Kedu samdi mo pay twa

\section{charlie}

Charlie O Charlie

Arrête de boire, arrête de boire le vin banane

Tu as gagné quinze sous et tu vas boire ton rhum

$\mathrm{Tu}$ vas ruiner la vie de cette pauvre femme

Dans le vin banane il y a toujours bêbêtes ciseaux (pinces oreilles)

Bêbêtes ciseaux vont te piquer la gorge

Charlie, Charlie arrête de boire du vin banane

Tu me fais crédit Kedu je te paierai samedi, je ne peux pas

Je vais essayer Kedu samedi je te paye, je ne peux pas

Laisse moi entrer Monsieur Kedu je te paierai samedi, je ne peux pas

Laisse moi essayer Kedu samedi je te paierai

Dans ce séga court et composé de plusieurs phrases injonctives, l'énonciateur interpelle Charlie et lui adresse quelques impératifs le sommant d'arrêter la boisson. Il argumente son discours et lui démontre qu'il cause sa perte ainsi que celle de sa femme en dépensant tout son argent. La forme de l'impératif ainsi tournée à l'affirmative exprime clairement l'ordre plutôt que le conseil ou la recommandation. Il s'agit d'une injonction plutôt que d'un conseil, visant alors à proscrire un certain type de comportement pour en exiger un autre.

Outre l'ordre et l'injonction, d'autres actes de langage plus subtils et moins directs visent le même dessein et peuvent être repérés dans le chant. Ces stratégies contribuent toutes à susciter l'adhésion et la complicité du public en même temps qu'elles ridiculisent et mettent à mal le personnage visé par la critique. L'intention comique se saisit aisément dans "Charlie », elle réside dans la dénonciation moqueuse d'une situation grotesque et pathétique. En effet, l'allusion aux bebet sizo qui pourraient piquer la gorge du malheureux s'il se mettait à boire la liqueur vient à coup sûr provoquer le rire chez les participants. Dans ce séga, la moquerie contribue à exclure moralement le comportement ciblé. De cette situation, se dessine le schéma typique de l'apologue qui fait suivre au récit une morale.

Dans les ségas de Ti Frèr, les paroles sont adressées aux participants par le biais de la mise en scène d'une fiction qui implique l'actualisation de personnages par le ségatierlocuteur. Les paroles s'adressent, non pas seulement aux personnages de la fiction, mais également à tout individu présent au moment de la performance. Les personnages mis en scène par le ségatier se situent entre le réel et la fiction, leurs traits sont inspirés de situations ayant existé, mais ils ne se réfèrent à aucun individu en particulier. Ils constituent plutôt des destinataires virtuels. Sous ce rapport, chaque membre de l'assemblée devient un destinataire potentiel de cette parole à la fois critique et injonctive. Le mode verbal du séga s'apparente ainsi à ce que Brigitte Desrosiers a pu identifier comme un "art de l'interpellation» (2004:258) puisque tout individu présent au moment de l'événement constitue un destinataire de la parole injonctive.

La fiction permet ainsi de créer des sortes de figures sous lesquelles sont exemplifiés certains types de comportements prohibés, comme la femme prise en faute ou bien l'alcoolique (qui peut également être incarné par une femme). Les personnages sont si simplifiés qu'ils constituent finalement une sorte de répertoire de stéréotypes facilement identifiables. La femme est une figure souvent associée à la faute dans les 
paroles de Ti Frèr ${ }^{20}$. Les ségas « Sa ti fam la », « Roseda », « Angeline » et « Fidelia » ${ }^{21}$ par exemple, témoignent de ces figures féminines constamment blâmées parce que leur comportement contredit un certain modèle domestique. "Angeline" montre par exemple une femme qui ne respecte pas ses devoirs d'épouse en cessant de préparer le repas à son mari alors que « Roseda » met en scène une femme qui préfère s'adonner à la boisson plutôt que de s'occuper de son enfant et de son mari qui revient du travail.

Ainsi, le recours à un système de communication fictif avec la constitution de figures stéréotypées permet de s'adresser à un public divers tout en évitant la confrontation avec un interlocuteur ciblé qui prendrait uniquement la critique pour lui-même (Desrosiers, 2004). Une telle communication constitue une alternative, un compromis discursif efficace dans cette pratique où la parole, définitivement ambiguë, balance entre le relâchement comique et l'expression coercitive.

\section{La construction poétique de la créolité}

Chanter la querelle dans le séga est synonyme d'une expression morale, pourtant celleci ne se réalise pas sans équivoque. La réprimande et l'ordre établi supposent toujours une inversion dans la dérision et la subversion. Des éléments qui tendent à rapprocher le séga de Ti Frèr du charivari dans sa dimension carnavalesque ${ }^{22}$.

L'action rituelle du séga de Ti Frèr opère au moyen d'antagonismes, la morale fonctionne grâce à la faute, le réel nourrit la fiction, le privé devient public, le blâme tient dans la dérision, et vice versa. Il y a en effet une ambiguïté inhérente à la pratique. Le paradoxe tient notamment au fait que des histoires tristes de la vie quotidienne - dans lesquelles se mêlent les sentiments de perte ou d'échec - sont chantées en même temps que s'exerce au cours de la performance le plaisir, l'ivresse et l'étourdissement, à travers la danse, la consommation d'alcool et les rencontres amoureuses $^{23}$. L'allégresse et l'hilarité, occasionnées par le renversement des identités ${ }^{24}$ et la moquerie, trouvent pour pendant la crainte et l'inquiétude d'être raillé à son tour. La pratique du séga qui excelle dans le chinage domestique est étroitement liée à des enjeux locaux et sociaux.

31 Les dialogues performés par Ti Frèr sont ainsi une occasion de mettre à bas des comportements - tels que la consommation d'alcool ou l'adultère - de montrer l'échec des attitudes des personnages interpellés, en somme de cristalliser au travers de ces situations le cadre de ce qui est permis et de ce qui est répréhensible. Sous ce rapport, les commentaires sarcastiques et hostiles viennent rappeler des impératifs de conduite et, à terme, ce que les individus devraient faire et comment ils doivent agir. Le séga de $\mathrm{Ti}$ Frèr constitue en ce sens un rituel collectif qui assure un pouvoir de contrôle informel du social. Dans «Fidelia» par exemple, les membres de l'assemblée assistent véritablement au châtiment symbolique de cette femme adultère - d'ailleurs nommée ironiquement Fidelia -, mais aussi à la mise en garde, au rappel à l'ordre des autres membres de la société quant aux pratiques adultères. Le conjoint semble ici dans son bon droit quand il s'agit de menacer sa femme de la battre, et son comportement à lui ne semble pas du tout poser problème. Les paroles de séga, du fait de leur libre expression, fournissent quelques informations sur les mœurs et le cadre de vie d'une partie de la communauté créole, très humble. Les portraits durs et caustiques chantés par Ti Frèr sont le témoignage d'une réalité vécue. L'alcoolisme de Roseda et la ruine de Charlie ne sont pas sans cause, ils résultent d'un lot de misères et d'une précarité au 
quotidien. Les situations relatées dans les paroles de séga révèlent, en filigrane, une souffrance et un malaise indéniables, ayant toujours en trame de fond la misère sociale. En effet, dans la micro-société rurale de Quartier Militaire ${ }^{25}$ relativement marginalisée des cadres officiels et dans laquelle très peu d'institutions marquantes sont représentées, la pratique du séga - comme modalité de contrôle social - se révèle symptomatique d'un malaise.

Les portraits que met en scène Ti Frèr dans ses ségas représentent trait pour trait ces images souvent dépeintes par les colons ou voyageurs occidentaux dans leurs écrits. Les caractéristiques des personnages reprennent en effet des définitions héritées de la colonisation esclavagiste ${ }^{26}$. Ces stéréotypes négatifs et humiliants, qui décrivent l'attrait des Créoles pour la boisson, leur incapacité à travailler ou à gérer un foyer, rejaillissent dans les paroles des ségas de Ti Frèr. Il est intéressant de constater que cette "figure du Créole" s'est perpétuée pour se reconfigurer dans les pratiques discursives.

Dans les ségas de Ti Frèr, le stéréotype colonial n'est pas repris tel quel, il est réintégré dans un contexte idéologique différent. Le stéréotype prend corps dans des personnages de fiction - véritables figures grotesques - et dont la caricature permet de désamorcer l'image humiliante. Par là, il s'agit de détourner des définitions exogènes dominantes pour se les approprier en imposant ses propres règles, à savoir, leur réintégration dans une logique autre. Ce processus de retournement du stigmate, qui exhibe ce qui est reproché, agit ainsi contre l'assignation de labels négatifs. Une telle démarche, qui vient briser "le miroir colonial de l'imaginaire" (Wolton, 2006, 255) s'inscrit en ce sens dans une réaction contrehégémonique, et cela dans un geste politique de réappropriation de sa propre image.

Le séga agit ainsi dans l'établissement et le maintien de certaines valeurs, lesquelles viennent renforcer le sentiment d'appartenance entre les membres. À ce titre, la mise en scène de séquences de la vie quotidienne invite à la construction d'un espace de partage et d'un imaginaire commun. Dans les ségas de Ti Frèr, la construction d'un ethos commun passe surtout par une communication des sentiments tristes. Les histoires de «Charlie » ou « Roseda » qui se noient, à cause de la misère engendrée par le chômage, dans la boisson ou encore celle de "Fidelia", enceinte d'une union illégitime et violemment menacée par son mari alors que celle-ci le supplie d'arrêter, témoignent d'une telle expression poétique. La tristesse, le regret ou la colère des personnages sont intensément exprimés dans les paroles, venant ainsi renforcer l'affection et les liens entre les membres qui assistent et participent au séga.

\section{Conclusion}

Les ségas de Ti Frer privilégient une communication chantée dramatisée plutôt qu'une forme de narration qui adresserait, à travers le seul acte de dire, des critiques ou des réprimandes à un interlocuteur ciblé (Desrosiers, 2004). L'interpellation critique et satirique s'effectue de manière indirecte à travers des scènes de fiction qui font intervenir des personnages incarnés par le ségatier-locuteur. Le destinataire des paroles de Ti Frèr est ainsi une figure, à la fois virtuelle et indéterminée, qui prend corps dans chacun des individus présents lors du séga.

L'idée que le séga de Ti Frèr constitue une modalité de contrôle social est une hypothèse pour comprendre la mise en scène de la querelle dans cette pratique poétique et 
musicale dansée. Il apparaît que celle-ci n'agit pas seulement selon des visées normatives et de moralisation des mœurs, elle révèle surtout les traces des expériences traumatiques de la colonisation et de l'esclavage sur la population mauricienne.

À l'aube de l'Indépendance, qui sera acquise en 1968 dans des circonstances violentes et difficiles, le ségatier Ti Frèr se fait l'écho de ce malaise, et cela, à travers l'expression poétique d'une subjectivité, d'un je qui se fait la voix de tous.

\section{BIBLIOGRAPHIE}

BORNAND, Sandra, 2010, Chants de douleur des femmes zarma du Niger, in Claude Calame, Florence Dupont, Bernard Lortat-Jacob, Maria Manca (éds), La voix actée. Pour une nouvelle ethnopoétique. Paris, Éditions Kimé.

BOSWELL, Rosabelle, 2006, Le malaise créole, ethnic identity in Mauritius, Oxford, New publications of Anthropology.

CARPOORAN, Arnaud, 2011, Le créole mauricien de poche, Paris, Assimil, 2011.

CHAZAN-GILLIG, Suzanne, RAMHOTA, Pavitranand, 2009, L'hindouisme mauricien dans la mondialisation Cultes populaires indiens et religion savante, Paris, Institut de Recherche pour le Développement, Khartala et MGI

CHILIN, Jérémy, 2009, Les Créoles dans les villes de l'île Maurice au milieu du vingtième siècle. Conflit ethnique à Port-Louis : les émeutes raciales de l'année 1968, Université Paris Diderot-Paris 7 [Mémoire de fin de deuxième cycle].

CoYAud, Maurice, 1980, La transgression des bienséances dans la littérature orale, Critique, $\mathrm{n}^{\circ} 394$, p. 325-332.

DÉODAT, Caroline, 2010, Le séga, un chant de transgression. Approche ethnopoétique d'une pratique poético-musicale dansée de l'île Maurice, Université Paris Diderot-Paris 7 [Mémoire de fin de deuxième cycle].

DESROSIERS, Brigitte, 2004, Le chant à l'île Rodrigues : rites, rires et sanctions, Kabaro II, 2-3, Diversité et spécificités des musiques traditionnelles de l'océan Indien, Paris, L'Harmattan.

Ducrot, Oswald, 1984, Le dire et le dit, Paris, Éditions de Minuit.

ENGLE MERRY, Sally, 1996, Rethinking gossip and scandal, in Daniel B. Klein (ed.), Reputation: Studies in the Voluntary Enforcement of Good Behavior, University of Michigan Press, pp. 47-74.

JAKOBSON, Roman, 1994, Essai de linguistique générale, les fondations du langage, Paris, Éditions de Minuit.

LE GOFF, Jacques et SCHMITT, Jean-Claude (dir.), 1981, Le charivari, Paris, École des Hautes Études en Sciences sociales et La Haye, Mouton.

PAIS DE BRITO, Joaquim, 2004, Motifs de tristesse, in Maurice Demeuldre (dir.), Sentiments doux-amers dans les musiques du monde, Paris, L'Harmattan, p. 217-222. 
RAUVILLE Hervé (de), 1909, « Artémidor » [chapitre XVI], L'île de France contemporaine, Paris, Nouvelle librairie nationale, p. 150-157.

RICAUD, Claudie, 1993, Le séga, re-création dans les traditions orales, Notre Librairie, $n^{\circ} 114$, p. $130-133$.

SERVAN-SCHREIBER, Catherine, 2010, Histoire d'une musique métisse à l'île Maurice, Chutney indien et séga Bollywood, Paris, Riveneuve éditions.

WOLTON, Dominique, 2006, Des stéréotypes coloniaux aux regards post-coloniaux : l'indispensable évolution des imaginaires, in Pascal Blanchard et Nicolas Bancel (dir.), Culture postcoloniale (1961-2006), traces et mémoires coloniales en France, Paris, Éditions Autrement.

ZUMTHOR, PAUL, 1983, Introduction à la poésie orale, Paris, Seuil.

\section{Discographie}

Hommage à Ti Frèr, Paris, Ocora Musique, Radio France, 1991.

\section{NOTES}

1. Dans sa création, le séga a mobilisé et s'est approprié divers apports tant africain, qu'européen et indien. Le séga est donc à penser comme le résultat d'un processus de création dynamique. Un processus qui continue d'opérer aujourd'hui, comme en témoigne « le séga Bollywood».

2. Les esclaves déportés sur la colonie viennent principalement de Madagascar et d'Afrique de l'est, et de façon minoritaire, de l'Inde.

3. Dans leur ouvrage L'hindouisme mauricien dans la mondialisation, Suzanne Chazan-Gillig et Pavitranand Ramhota précisent en effet que «la religion et les différences culturelles d'origine ont été les facteurs principaux des différenciations sociales » à l'île Maurice $(2009,12)$.

4. "In Mauritius, however, everyone who is not of pure French, British, Indian or Chinese blood comes under the term Creole, although these people are divided into two groups with separate identities: the coloured communities of mixed European-African-Indian descent. The community of African origin whose descendants gained their freedom when slavery are abolished" (Mannick cité par Chilin, 2009, 11).

5. La situation des Créoles s'explique en grande partie par près de trois siècles de colonisation qui affecta très profondément cette population. $\mathrm{Au} \mathrm{xx}^{\mathrm{e}}$ siècle, la communauté créole se distingue en deux catégories sociales. La première constitue une élite dite «de couleur » au sein de la société mauricienne, elle se situe principalement dans les zones urbaines comme Curepipe, RoseHill, Beau-Bassin et la capitale Port-Louis. La seconde désigne une catégorie de population nommée les «Ti Créoles ». Ce groupe reste relativement à l'écart des centres urbains et se situe dans les zones rurales côtières et les plateaux, où ses membres exercent des emplois précaires comme dockers, coupeurs de cannes, artisans ou encore commis (Chilin, 2009).

6. Jean Alphonse Ravaton, surnommé Ti Frèr, est né en 1900 à Quartier Militaire.

7. Cette manifestation appelée la "Nuit du séga » est organisée par l'office du tourisme le 24 octobre 1964. Elle se tient au pied de la montagne du Morne, à Rivière Noire - région historiquement symbolique, où s'installèrent autrefois des esclaves «marrons ", puis d'autres, nouvellement libres. La "Nuit du séga», en tant que première représentation officielle, correspond au moment historique où cette pratique, autrefois marginalisée, devient en quelque sorte affaire publique. Par la suite, le séga va suivre un mouvement de centralisation, quittant partiellement les milieux ruraux et les côtes pour les centres urbains.

8. Véritable emblème de cette pratique à partir des années 1965, Ti Frèr est souvent désigné dans les médias comme « le Père du séga». 
9. Les situations de la vie quotidienne servent à inspirer le ségatier qui les utilise pour construire les histoires qu'il chante dans ses ségas. Ainsi, il reprend certains prénoms d'individus de son entourage. Papitou était par exemple un membre de sa famille. Il utilise aussi des scènes dont il a été témoin comme point de départ à la fiction, un dialogue auquel il a assisté dans un bateau est ainsi repris dans le séga «Charlie» (Déodat, 2010, 28). Dans les ségas de Ti Frèr, le réel sert de support à la fiction, et inversement.

10. À l'instar de Paul Zumthor, je prends le terme de "performance » dans son acception anglosaxonne. Zumthor définit la performance comme une « action complexe par laquelle un message poétique est simultanément transmis et perçu, ici et maintenant. Locuteur, destinataire(s), circonstances se trouvent concrètement confrontés, indiscutables » $(1983,32)$.

11. Les instruments du séga de Ti Frer sont la ravane (tambour plat à peau de chèvre), la maravane (caisse plate de forme rectangulaire enfermant des pois), le triangle et l'accordéon diatonique.

12. «Il [le séga-laé] a en général une structure tri-partite. La première partie est le plus souvent instrumentale, avec les ravanes en premier plan. Pendant cette partie, les ravaniers jouent des cellules rythmiques qui alternent entre celles caractéristiques de l'attente et de l'appel au chanteur. (...) La deuxième partie commence dès que le chanteur accepte cet appel instrumental et $\mathrm{y}$ répond à travers le chant. Cette partie est la plus importante textuellement, car c'est à ce moment que l'histoire de la chanson nous sera révélée. (...) Dans la troisième partie, que beaucoup de musiciens appellent la roulade, le chanteur entrecoupe le texte, déjà utilisé dans la partie précédente, de monosyllabes, "ééla- é-la-é ", et de manière générale, il n' y a pas de nouveauté textuelle présentée dans cette partie. En fait, cette dernière partie appartient surtout aux danseurs qui, jusque-là, étaient présents, mais auditeurs uniquement. » (Ricaud, 1993, 130).

13. Le final correspond à la dernière figure exécutée dans le quadrille. Le séga final privilégie l'utilisation de l'accordéon alors que le séga-laé n'en fait pas usage dans ses compositions, mais adopte davantage le triangle. Le séga final constitue, par la fonction plus harmonique de l'accordéon, une forme plus mélodique, alors que le séga-laé semble plus rythmique du fait de l'utilisation d'instruments à percussion: le triangle et la maravane (idiophones) et la ravane (membranophone).

14. Notons que dans le créole mauricien, les sons [z] et [s] se substituent aux sons [ch] et [j]. On dira par exemple "zenes" en créole mauricien plutôt que "jeunesse » en français. Cette particularité linguistique confère ainsi au créole mauricien des qualités sonores plus sifflantes.

15. "In these dances, the top part of the body stays relatively immobile while the hips moves." (Boswell, 2006, 61).

16. Le corps doit être conçu « comme un objet et une réalité physique et sociale » (Pais de Brito, 2004, 218) permettant de penser les aspects esthétiques et musicaux de cette pratique. Il porte en lui les stigmates de la pauvreté et de l'exclusion, les imperfections causées par l'alcool et la mauvaise dentition, qui s'impriment dans les manières de chanter et de danser. Le corps subit encore l'effet de la censure puisque la gestuelle de la danse du séga, jugée obscène, était rejetée par les représentants de l'Église.

17. «Le locuteur, responsable de l'énoncé, donne existence, au moyen de celui-ci, à des énonciateurs dont il organise les points de vue et les attitudes» (Ducrot, 1984, 205).

18. Voir les transcriptions de ces ségas ici même.

19. Nous avons défini que la fonction prédominante du message du séga était la fonction conative. Orientée vers le destinataire, elle «trouve son expression grammaticale la plus pure dans le vocatif et l'impératif » (Jakobson, 1994, 216).

20. Les stéréotypes qui se dessinent dans les paroles de Ti Frèr s'apparentent à des stéréotypes négatifs, sociaux ou de genre: la figure du créole et la femme fautive. Voir plus bas: «La construction poétique de la créolité ». 
21. Les ségas «Sa ti fam la » et « Angeline » sont accessibles sur le CD Hommage à Ti Frèr (1991) ainsi que les transcriptions des paroles (Déodat, 2010).

22. Le séga s'apparente au charivari à bien des égards: dans la ritualisation des pratiques discursives, dans les processus d'inversion et dans les intentions de moralisation des mœurs (domestiques et sexuelles). Dans les ségas de Ti Frèr, la morale et la faute agissent dans une réversibilité constante, car à tout moment, la faute est susceptible de rejoindre la norme pour mettre en marge un certain type de comportement.

23. Dans les ségas de Ti Frèr, les antagonismes sont provisoires. Chacun tient son rôle durant l'action rituelle, mais le personnage tend à s'évanouir, au-delà de la fiction. En effet, le séga se déroulait dans une ambiance particulièrement échauffée, surtout du fait de la consommation d'alcool.

24. Le ségatier Ti Frèr opère des changements de rôles parfois de manière consécutive dans un même séga. Dans le séga "Fidelia», le renversement des identités implique une inversion de genre et de statut, puisque Ti Frèr incarne successivement Fidelia puis son mari.

25. La localité de Quartier-Militaire se caractérise par sa ruralité et par sa mixité ethnique. Elle comprend une majorité d'Indo-mauriciens et une minorité de Créoles.

26. «Le travail quotidien était pour eux [les esclaves] une salutaire habitude qu'ont perdue, hélas leurs descendants. (...) Quel usage le noir a-t-il fait de cette liberté ? Le premier soin des esclaves libérés a été d'abandonner complètement la culture de la terre, comme indigne de leur nouvelle condition. (...) Artémidor, ne voulant plus être laboureur, s'est fait une spécialité des métiers manuels il est charpentier, maçon, cordonnier, tailleur, etc. (...) il n'a ni l'activité ni l'intelligence de l'Indien et s'est créé des besoins en disproportion avec sa position (...) la vanité leur fit tant donner au luxe et la paresse au chômage, qu'aujourd'hui, après trois générations à peine, ils ne possèdent plus que deux petites cases entourées d'un carré de terre insignifiant (...) C'est, hélas l'histoire de la plupart de ces familles d'anciens esclaves. » (de Rauville, 1909, 154-157).

\section{RÉSUMÉS}

Ti Frèr, figure désormais emblématique du séga mauricien $\mathrm{du} \mathrm{xx}^{\mathrm{e}}$ siècle, chante le quotidien et la vie des Créoles à travers des séquences de querelle. Dans les paroles, chantées par le ségatier, la critique et les commentaires hostiles sont dirigés à l'égard des participants à travers une mise en scène de fiction. Les personnages s'interpellent sur des faits du quotidien, qui vont de la conquête amoureuse à la dispute conjugale, de la misère du chômage à celle de l'alcoolisme. Suivant une approche ethnopoétique et une analyse anthropologique de la poésie, nous démontrons dans cet article que le séga constitue une modalité de contrôle social. Les paroles de séga, prises dans un système de communication polyphonique viennent dicter de manière indirecte des règles de bonne conduite à l'ensemble de la communauté présente lors du rituel.

A major figure in the xxth century Mauritian sega, Ti Frer evokes the everyday life of Creoles through quarrel sequences. In the lyrics, sung by the speaker, criticisms and admonitions are directed to the audience's members through the use of mise-en-scène. Characters shout at one another about the details of everyday life, ranging from love conquest to relationship problems, from unemployment misery to alcoholism and violence. Using an ethnopoetical approach and an anthropological analysis of poetry, we demonstrate in this article that Ti Frer's segas establish a 
modality of social control within the Creole group. Segas' lyrics, when considered in a polyphonic communication system, turn out to be an indirect way to dictate rules of good behavior to the members of the society.

INDEX

Thèmes : anthropologie (Océanie), ethnopoétique

Mots-clés : Séga, communication indirecte, malaise créole

Keywords : Sega, Mauritius, Ethnopoetic, Indirect Communication, Creole Predicament, Anthropology

Index géographique : Maurice (île) 